\title{
HDAC inhibitor apicidin suppresses murine oral squamous cell carcinoma cell growth in vitro and in vivo via inhibiting HDAC8 expression
}

\author{
MEE-YOUNG AHN \\ Department of Pharmaceutical Engineering, Division of Bio-Industry, College of Medical and Life Sciences, \\ Silla University, Busan 46958, Republic of Korea
}

Received April 4, 2018; Accepted August 23, 2018

DOI: $10.3892 / 01.2018 .9468$

\begin{abstract}
Apicidin, a cyclic peptide histone deacetylase (HDAC) inhibitor, has been demonstrated to exhibit antitumor activity in a number of human cancer types. The present study examined the antitumor activity of apicidin in murine oral squamous cell carcinoma (OSCC) cells. Inhibition of cell proliferation and the expression of selective HDACs were determined in apicidin-treated AT-84 murine OSCC cells. A C3H mouse model with subcutaneous injection of AT-84 cells was used to assess the in vivo effect of apicidin on tumor growth. Apicidin-induced cell growth inhibition and selectively reduced HDAC8 expression in AT-84 cells. Induction of apoptosis and autophagy was observed in apicidin-treated AT-84 cells. Apicidin notably inhibited tumor growth by up to $46 \%$ relative to the control group at the end of a 14-day period in a murine tumor model. The immunohistochemistry results in tumor tissues indicated that apicidin inhibited cell proliferation and induced apoptosis and autophagy in AT-84 cell-derived tumor tissues. Overexpression of HDAC8 was observed in the nucleus and cytoplasm in tumor tissues and apicidin significantly inhibited the level of HDAC8 expression, compared with the vehicle group. These results indicated that apicidin inhibited cell proliferation through HDAC8 inhibition in murine OSCC cells in vitro and in vivo. The present study indicated that apicidin may be an effective therapeutic agent for OSCC.
\end{abstract}

\section{Introduction}

Epigenetic regulation alters the gene expression through chromatin structural modification without DNA sequence

Correspondence to: Professor Mee-Young Ahn, Department of Pharmaceutical Engineering, Division of Bio-Industry, College of Medical and Life Sciences, Silla University, 700 Beon Road, Busan 46958, Republic of Korea

E-mail:myahn@silla.ac.kr

Key words: apicidin, histone deacetylase 8, apoptosis, autophagy, murine oral squamous cell carcinoma changes (1). Post-translational modifications of histones, including acetylation, serve an important role in regulating gene expression (2). The pattern of histone acetylation is determined by two groups of enzymes: Histone acetyltransferases and histone deacetylases (HDACs). The deacetylation of lysine residues by HDAC is associated with condensed chromatin state and transcriptional gene silencing (3). HDACs alter the transcription of genes that regulate essential cell functions, including cell growth, cell cycle regulation and apoptosis (1). HDACs have been subdivided into a family of 18 genes that represent four classes: Class I (HDAC1, 2, 3 and 8), Class II (HDAC4, 5, 6 and 11), Class III (HDAC11) and Sirtuins (Sirt1-7). Upregulation of HDACs has been determined in numerous human cancer types, including oral cancer $(1,4,5)$. Therefore, HDACs have become an attractive target for cancer therapeutics.

HDAC inhibitors have emerged as a novel class of anticancer drugs. They are structurally classified into at least four groups: Hydroxamic acids, short-chain fatty acids, benzamides and cyclic peptides $(6,7)$. These agents vary notably in their potency and specificity toward individual HDACs (8). Apicidin is a cyclic peptide isolated as a fungal metabolite from fermentations of Fusarium spp (9). Apicidin has been reported to exhibit a proliferative effect in various cancer types, including leukemia, ovarian cancer and hepatocellular carcinoma (10-12). Apicidin primarily induces cell cycle arrest and apoptosis through caspase activation in cancer cells (10-12). However, specific targets of apicidin in a variety of cancer types, including lung and pancreatic cancer, remain unclear, and research into the molecular mechanism of apicidin for anticancer activity remains ongoing in pre-clinical studies (13-16).

Oral cancer is a group of neoplasms located in the oral cavity, pharyngeal regions and salivary glands (17). Oral squamous cell carcinoma (OSCC) is the most common oral cancer type and accounts for $>90 \%$ of human oral malignancy types (18). OSCC is frequently treated with a combination of surgery, radiotherapy and chemotherapy (19). Despite advanced therapeutic approaches, the incidence and mortality rates for OSCC have not significantly improved in the past 30 years (17); therefore, improving the treatment outcome for OSCC requires investigation into novel therapeutic strategies. Our previous study demonstrated that the HDAC inhibitor 
apicidin exerts anti-proliferative effects on human OSCC cell lines (20). However, the members of HDACs that are selectively inhibited by apicidin remain unclear, and in vivo antitumor efficacy has not been examined in OSCC. Identification of an isoform selective HDAC inhibitor may improve the therapeutic potential and reduce the cytotoxicity associated with cancer treatment. Therefore, the present study aimed to examine the selective HDAC inhibitory effect of apicidin in vitro and in vivo, and the in vivo antitumor effect of apicidin, in a murine OSCC model.

\section{Materials and methods}

Cell culture and chemicals. The murine OSCC AT-84 cells were provided by Dr E. J. Shillitoe (Upstate Medical University, Syracuse, NY, USA) (21). AT-84 cells originated from a spontaneous murine $\mathrm{SCC}$ in the oral mucosa of $\mathrm{C} 3 \mathrm{H}$ mice (22) and were isolated by Hier et al (23). The cells were maintained in RPMI-1640 medium containing 10\% fetal bovine serum (Gibco; Thermo Fisher Scientific, Inc., Waltham, MA, USA) and $100 \mathrm{U} / \mathrm{ml}$ penicillin-streptomycin (Welgene, Inc., Daegu, Korea) at $37^{\circ} \mathrm{C}$ in an atmosphere containing $5 \% \mathrm{CO}_{2}$. Unless stated otherwise, all chemicals were purchased from Sigma-Aldrich (Merck KGaA, Damstadt, Germany). Apicidin (Sigma-Aldrich; Merck KGaA) was dissolved in sterile DMSO to generate a $5 \mathrm{mM}$ stock solution, which was stored at $-80^{\circ} \mathrm{C}$. The cells were treated with culture media alone as a control, or with various concentrations $(0.1,0.5,1,5$ or $10 \mu \mathrm{M})$ of apicidin (the maximum final concentration of DMSO was $<0.1 \%$ ) for $24 \mathrm{~h}$.

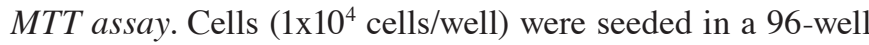
plate and incubated overnight to allow attachment. Cells were treated with apicidin at the aforementioned concentrations for $24 \mathrm{~h}$. At the end of the treatment period, $10 \mu \mathrm{l}$ MTT (Sigma-Aldrich; Merck KGaA) reagent $(5 \mathrm{mg} / \mathrm{ml})$ was added to each well (final concentration, $0.5 \mathrm{mg} / \mathrm{ml}$ ). After $4 \mathrm{~h}$ at $37^{\circ} \mathrm{C}$, the supernatant was aspirated and formazan crystals were dissolved in $100 \mu 1$ DMSO. A microplate autoreader ELISA was used to determine the absorbance at $595 \mathrm{~nm}$. All experiments were performed in triplicate.

Western blot analysis. The cells were washed with PBS and harvested in a lysis buffer (Intron Biotechnology, Inc., Seongnam, Korea). Protein concentrations were measured using a Bradford protein assay kit, according to the manufacturer's protocols. Samples containing equal amounts of protein $(50 \mu \mathrm{g})$ were resolved on SDS-PAGE in a $10-15 \%$ gel and transferred to a polyvinylidene difluoride membrane. Following blocking with 5\% skim milk in tris-buffered saline with $0.1 \%$ Tween-20 (TBS-T) for $1 \mathrm{~h}$ at room temperature, the membranes were incubated with primary antibodies $(1: 1,000$ dilution) against acetylated histone $\mathrm{H} 4$ (cat. no. 07-108; Upstate Biotechnology, Inc., Lake Placid, NY, USA), HDAC8 (cat. no. ab187139; Abcam, Cambridge, MA, USA), HDAC7 (cat. no. SC-11421; Santa Cruz Biotechnology, Inc., Dallas, TX, USA), HDAC1 (cat. no. 5356), HDAC2 (cat. no. 5113), HDAC4 (cat. no. 7628), HDAC6 (cat. no. 7612), cleaved caspase-3 (cat. no.9664), poly(ADP-ribose) polymerase (PARP; cat. no. 9542), microtubule associated protein 1 light chain 3B (LC3B; cat. no. 3868), autophagy-related protein 7 (ATG7; cat. no. 2631), p62 (cat. no. 5114; Cell Signaling Technology, Inc., Danvers, MA, USA) and $\beta$-actin antibody (cat. no. SC-47778; Santa Cruz Biotechnology, Inc.) overnight at $4^{\circ} \mathrm{C}$. The membranes were then washed six times with TBS-T and incubated with horseradish peroxidase-conjugated secondary antibodies (anti-mouse, cat. no. SC-2354; anti-rabbit, cat. no. SC-2768; 1:5,000 dilution; Santa Cruz Biotechnology, Inc.) for $1 \mathrm{~h}$ at room temperature. Finally, the membranes were washed six times with TBS-T and visualized using the Pierce ECL western Blotting Substrate (cat. no. 32209; Thermo Fisher Scientific, Inc.) on an ATTO chemiluminescent imaging system (ATTO Corporation, Tokyo, Japan).

Flow cytometry analysis for apoptosis detection. The cells were stained using a Alexa Fluor ${ }^{\circledR} 488$ Annexin V/Dead cell Apoptosis kit with Alexa Fluor ${ }^{\circledR} 488$ Annexin V and propidium iodide (PI; cat. no. V13241; Molecular Probes; Thermo Fisher Scientific, Inc.), according to the manufacturer's protocol for apoptosis analysis. A Cell Lab Quanta ${ }^{\mathrm{TM}} \mathrm{SC}$ flow cytometer (Beckman Coulter Inc., Brea, CA, USA) with Cell Lab Quanta SC software (version 1.0; Beckman Coulter Inc.) were used to perform data acquisition and analysis.

Detection of acidic vesicular organelles (AVOs). Autophagy is characterized by the formation and promotion of AVOs. To detect the development of AVOs, the cells were stained with acridine orange, as described previously (20). In acridine orange-stained cells, the cytoplasm and nucleus fluoresce bright green, whereas acidic compartments fluoresce bright red. The treated cells were stained with acridine orange ( $1 \mu \mathrm{g} / \mathrm{ml}$; Sigma-Aldrich; Merck KGaA) for $15 \mathrm{~min}$ at $37^{\circ} \mathrm{C}$. To quantify the development of AVOs, the increased red stained cells were analyzed using a FACScan flow cytometer and CellQuest Pro software (version 4.0; BD Biosciences, San Jose, CA, USA).

Autophagy inhibition and trypan blue exclusion assay. A specific autophagy inhibitor chloroquine (CQ; $50 \mu \mathrm{M}$; Sigma-Aldrich; Merck KGaA) was co-treated with apicidin (Sigma-Aldrich; Merck KGaA) in AT-84 cells for $24 \mathrm{~h}$ at $37^{\circ} \mathrm{C}$. Following treatment, cell proliferation was measured using a trypan blue exclusion assay. The trypan blue exclusion assay was based on the capability of viable cells to exclude the dye. After 5 min of treatment of cells with $0.4 \%$ trypan blue (Sigma-Aldrich; Merck KGaA) at room temperature, they were loaded into a hemocytometer and counted for the dye uptake. The number of viable cells was calculated as the percentage of the total cell population.

In vivo study on AT-84 cells bearing $\mathrm{CH} 3$ mice. AT-84 cells were used for the in vivo experiments, previously established by Pang et al (24). Male C3H mice (6 weeks old, 20-22 g) were purchased from Samtaco Bio Korea (Osan, Korea). All animals were housed under controlled temperature $\left(22 \pm 2^{\circ} \mathrm{C}\right)$ and relative humidity (50-60\%) during a $12 \mathrm{~h}$ light-dark cycle. The animals were provided food and tap water ad libitum. Mice were inoculated subcutaneously in the right flank with $1 \times 10^{7}$ AT- 84 cells. When the tumor size reached $150-200 \mathrm{~mm}^{3}$, the mice were divided randomly into two groups, with six mice/group. Apicidin $(5 \mathrm{mg} / \mathrm{kg}$ ) or vehicle control 


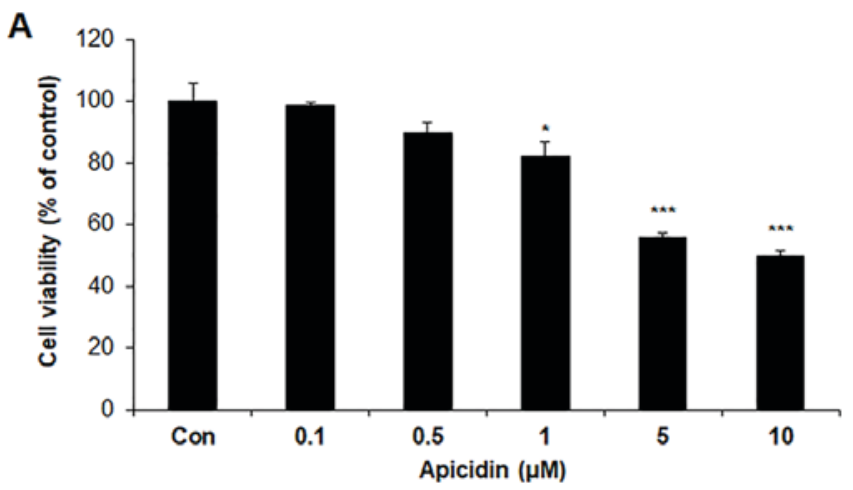

B

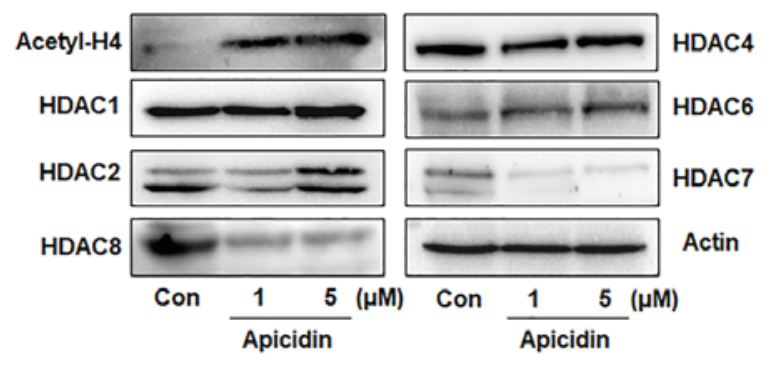

Figure 1. Growth inhibition and the expression of HDAC in apicidin-treated AT-84 cells. Cells were incubated with the indicated concentrations of apicidin for $24 \mathrm{~h}$. (A) The levels of cell proliferation were measured with an MTT assay. The data are reported as the mean \pm standard deviation of three independent experiments. ${ }^{*} \mathrm{P}<0.05$ and ${ }^{* * *} \mathrm{P}<0.001$, compared with the control. (B) Changes in acetylation-associated protein levels were analyzed with western blot analysis using antibodies against Actyl-H4, and HDAC1, 2 , 4, 6, 7 and 8 . The protein levels were normalized to actin levels. The representative bands from three independent experiments are depicted. Acetyl-H4, acetylated histone H4; HDAC, histone deacetylase 8; Con, control.

(0.1\% DMSO) was administered to the mice intraperitoneally every other day for 14 days. The animals were monitored daily and tumor volume was measured with calipers and calculated with the formula: $\left(a b^{2}\right) / 2$, where $a$ is the longest diameter and $\mathrm{b}$ is the shortest diameter of the tumor. The mice were sacrificed at the end of the treatment period. The tumor tissue was excised, weighed and fixed in $4 \%$ paraformaldehyde at room temperature over $24 \mathrm{~h}$. All experiments were performed under protocols approved by the Ethical Committee of Animal Care and Use at Pusan National University Hospital (Busan, Korea; approval no. PNUH-2016-090).

Histopathology. The animals were sacrificed on day 15, and the tumors were carefully removed and fixed in $4 \%$ paraformaldehyde at room temperature over $24 \mathrm{~h}$. The tissues were embedded in paraffin wax and cut into $2 \mu \mathrm{m}$ sections. The slides were de-paraffinized with xylene and rehydrated a graded alcohol series (100, 90, 70 and 50\% ethyl alcohol) for $10 \mathrm{~min}$ at room temperature, and stained with $10 \%$ hematoxylin (Vector Laboratories, Inc., Burlingame, CA, USA) for $5 \mathrm{~min}$ and $0.5 \%$ eosin for $1 \mathrm{~min}$ at room temperature for histological examination. The result was viewed under a light microscope at magnification, x200.

Immunohistochemistry and terminal deoxynucleotidyl transferase-mediated dUTP nick end labeling (TUNEL) assay. The sections were incubated in $3 \% \mathrm{H}_{2} \mathrm{O}_{2}$ in $70 \%$ methanol for
$10 \mathrm{~min}$ to remove endogenous peroxidase. Subsequently, they were blocked with $1 \%$ bovine serum albumin (Sigma-Aldrich; Merck KGaA) in PBS for $1 \mathrm{~h}$ at room temperature. Following this, the sections were incubated with proliferating cell nuclear antigen (PCNA; 1:100 dilution; cat. no. M0879; Dako, Carpinteria, CA, USA), LC3B (1:100 dilution; cat. no. 3868; Cell Signaling Technology, Inc.), p62 (1:100 dilution; cat. no. 5114; Cell Signaling Technology, Inc.) and HDAC8 (1:100 dilution; cat. no. ab217702; Abcam) antibodies overnight at $4^{\circ} \mathrm{C}$. After washing three times with PBS-T, the sections were subjected to the avidin-biotin peroxidase complex (ABC) method using universal an $\mathrm{ABC}$ kit with biotinylated horse anti-mouse IgG/rabbit IgG secondary antibodies and ABC reagent (R.T.U. VECTASTAIN ${ }^{\circledR}$ Universal Elite ABC kit; cat. no. PK-7200; Vector Laboratories, Inc.), according to the manufacturer's protocol. Additionally, peroxidase activity was evaluated with 3,3'-diaminobenzidine (DAB Peroxidase Substrate kit; cat. no. SK-4100; Vector Laboratories, Inc.). Finally, 10\% hematoxylin for $1 \mathrm{~min}$ at room temperature was used to counterstain the sections. The TUNEL assay was performed using an TdT DAB In Situ Apoptosis Detection kit (cat. no. 4810-30-k; R\&D Systems, Inc., Minneapolis, MN, USA), according to the manufacturer's protocol. The result was viewed under a light microscope at magnification, $x 200$ and $\times 400$. The positive cells were counted from five randomly selected areas under magnification, $\mathrm{x} 400$ and represented the mean \pm standard deviation.

Statistical analysis. Data are expressed as the mean \pm SD of at least three individual experiments. Statistical analysis was performed using statistical software SPSS (version 20; IBM Corp., Armonk, NY, USA). Statistical differences among groups were performed with an one-way analysis of variance followed by Scheffe's post-hoc test. Differences between groups were determined with a Student's t-test. $\mathrm{P}<0.05$ was considered to indicate a statistically significant difference.

\section{Results}

Effect of apicidin on AT-84 cell viability and HDAC expression. In order to analyze the effects of apicidin on cell viability, AT-84 cells were treated with increasing concentrations of apicidin and the cell viability was assessed with an MTT assay. Apicidin inhibited the viability of AT- 84 cells in a dose-dependent manner, whereby concentrations of $\geq 1 \mu \mathrm{M}$ resulted in a significant reduction (Fig. 1A). Subsequently, the effect of apicidin on the levels of histone acetylation and specific HDACs was analyzed using western blotting. The cells were treated with the indicated concentrations of apicidin (1 and $5 \mu \mathrm{M}$ ) for $24 \mathrm{~h}$. As depicted in Fig. 1B, apicidin treatment notably increased the level of acetylated histone $\mathrm{H} 4$ and decreased the level of HDAC8 in AT-84 cells. HDAC7 expression was also marginally reduced, but there was no change in the expression of other HDACs in apicidin-treated AT-84 cells. These results indicated that apicidin induces cell growth inhibition and that it primarily inhibited HDAC8 expression in AT-84 cells.

Apoptosis induction on apicidin-treated AT-84 cells. To elucidate the mechanism underlying the reduction in viability of AT-84 cells following treatment with apicidin, apoptosis was 
A

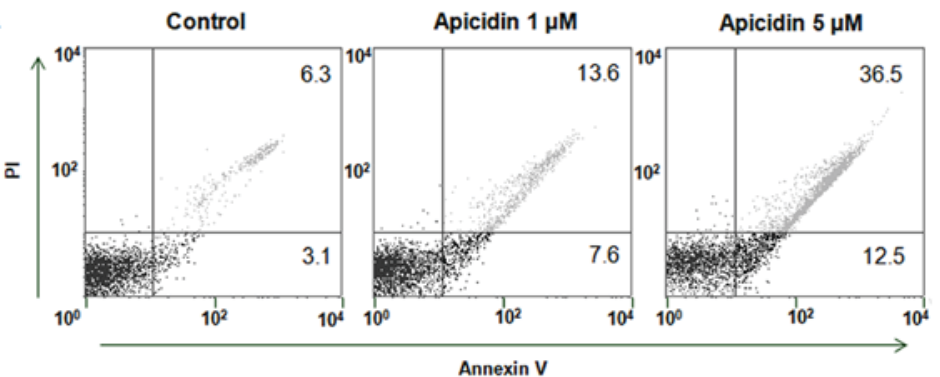

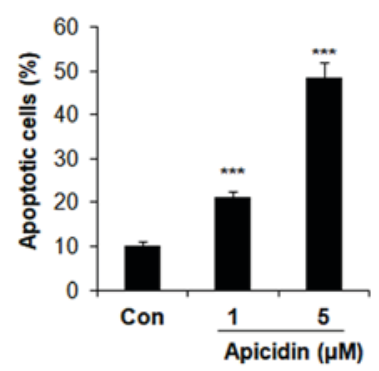

C

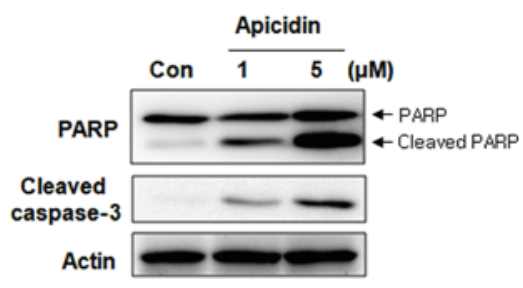

Figure 2. Induction of apoptosis in apicidin-treated AT-84 cells. AT-84 cells were incubated with 1 or $5 \mu \mathrm{M}$ apicidin for 24 h. Apoptosis was evaluated by Annexin V and PI staining with flow cytometry. (A) Representative images and (B) quantification of flow cytometric results. The data are reported as the mean \pm standard deviation of three independent experiments. ${ }^{* * *} \mathrm{P}<0.001$, compared with the control. (C) The expression levels of PARP and cleaved caspase-3 were detected with western blot analysis. The protein levels were normalized with a comparison with actin levels. The representative bands from three independent experiments are depicted. PARP, poly(ADP-ribose) polymerase; Con, control; PI, propodium iodide.

measured with an Annexin V apoptosis assay. AT-84 cells were treated with 1 and $5 \mu \mathrm{M}$ apicidin for $24 \mathrm{~h}$, and Annexin-V/PI double staining was examined with flow cytometry. Apicidin significantly increased the percentage of Annexin V-positive apoptotic cells in a dose-dependent manner compared with the untreated control group (Fig. 2A and B). The ratio of Annexin V-positive cells was 21 and $49 \%$ at doses of 1 and $5 \mu \mathrm{M}$ apicidin, respectively compared with $6.3 \%$ in the control group. The expression of apoptosis-associated protein levels was measured with western blot analysis to confirm apoptosis involvement. As depicted in Fig. 2C, a concentration-dependent increase in cleaved PARP and cleaved caspase- 3 expression levels were observed in apicidin-treated AT-84 cells, whereas levels of total PARP remained constant. These results indicated that apicidin induced caspase-dependent apoptosis in AT-84 cells.

Autophagy induction on apicidin-treated AT-84 cells. To determine whether apicidin induces autophagy in AT-84 cells, acridine orange staining was performed and evaluated with flow cytometry. As depicted in Fig. 3A, red fluorescence intensity in flow cytometric analysis was dose-dependently increased with apicidin treatment in AT-84 cells, indicating an enhancement of AVOs. The ratio of red fluorescence intensity was 24 and $78 \%$ at doses of 1 and $5 \mu \mathrm{M}$ apicidin, respectively, both of which was significantly increased compared with the untreated control group (Fig. 3B). Autophagy-associated protein expression was measured using western blotting to confirm the induction of autophagy. Apicidin notably induced the expression level of ATG7 and LC3B-II, a specific marker of autophagosome promotion (Fig. 3C). The level of p62, a marker for autophagic flux, was decreased in apicidin-treated AT-84 cells. Additionally, to determine whether autophagy by apicidin contributes to survival or death, AT- 84 cells were co-treated with apicidin and CQ, a specific autophagy inhibitor. Cell viability was examined using the trypan blue exclusion assay. As depicted in Fig. 3D, apicidin with CQ treatment significantly reduced cell viability, compared with apicidin without CQ. These results demonstrated that apicidin induced autophagy in AT-84 cells and that autophagy induction by apicidin may be associated with cell protection.

In vivo antitumor effect on apicidin treatment. The in vivo effect of apicidin on tumor growth was examined using mice inoculated with AT- 84 cells. These mice were administered with apicidin or vehicle every other day for a 14-day period. As depicted in Fig. 4A, apicidin significantly decreased the tumor volume, compared with the control group, and inhibited tumor growth up to $47 \%$ relative to the control group at the end of the 14-day period. Mean tumor weight was also significantly reduced in the apicidin-treated group, compared with the vehicle-treated control group (Fig. 4B). No significant systemic toxicity, including body weight changes or other apparent adverse effects, was observed in the animals throughout the study period (Fig. 4C). These results indicated that apicidin exhibited an antitumor effect against AT-84 cells in vivo.

Histological changes and immunohistochemistry analysis. The histological changes between apicidin-treated and control groups were examined using hematoxylin and eosin staining in paraffin-embedded tumor sections. Markedly increased eosinophilic cytoplasm and nuclear changes were observed in tumor tissues treated with apicidin, compared with the control tumor tissues (Fig.5). Subsequently, the immunohistochemistry was examined with regard to the antitumor effects of apicidin in tumor tissues. As depicted in Fig. 5A and C, tumor tissues treated with apicidin exhibited significantly decreased PCNA and HDAC8 expression levels, compared with control tumor 

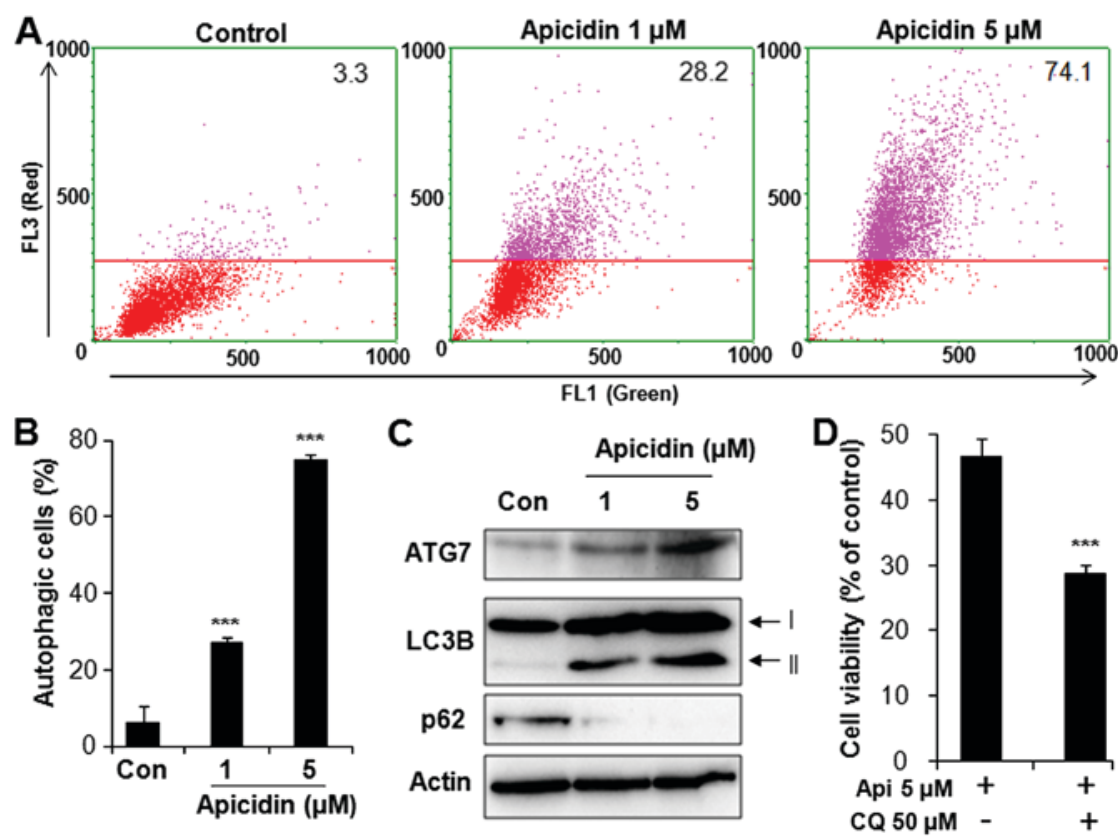

Figure 3. Induction of autophagy in apicidin-treated AT- 84 cells. AT-84 cells were incubated with 1 or $5 \mu \mathrm{M}$ apicidin for 24 h. Autophagy was evaluated by acridine orange staining with flow cytometry. (A) Representative image and (B) quantification of flow cytometric results. The value of red positive cells (the number in the upper right) indicates percentage of autophagic cells. The data are reported as the mean \pm standard deviation of three independent experiments. ${ }^{* * *} \mathrm{P}<0.001$, compared with the control. (C) The expression levels of LC3B-II, ATG7 and p62 were detected by western blot analysis. The protein levels were normalized to actin levels. Representative bands from three independent experiments are depicted. (D) Cells were treated with $5 \mu \mathrm{M}$ apicidin for $24 \mathrm{~h}$ in the absence or presence of CQ $(50 \mu \mathrm{M})$. Cell viability was determined using a trypan blue exclusion assay following co-treatment with apicidin and CQ ${ }_{* * * *} \mathrm{P}<0.001$, compared with the only apicidin-treated group. Con, control; Api, apicidin; ATG7, autophagy-related protein 7; LC3B, microtubule associated protein 1 light chain $3 \mathrm{~B} ; \mathrm{CQ}$, chloroquine.

A

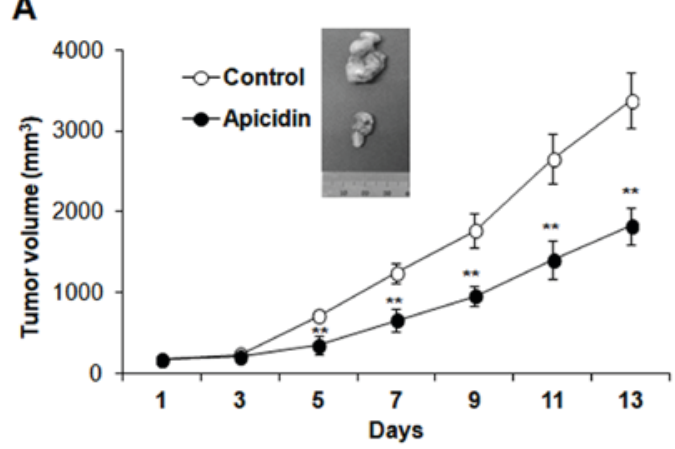

C
B

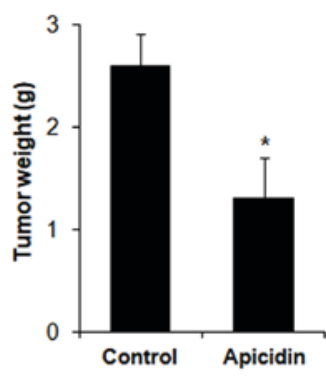

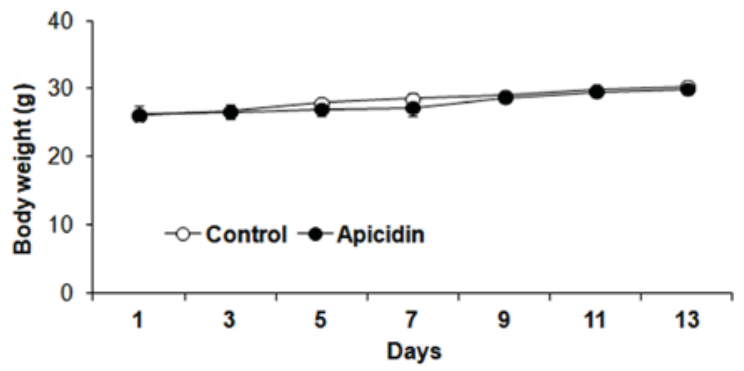

Figure 4. Effect of apicidin on the growth of AT- 84 cell-derivated allografts. AT- 84 cells $\left(1 \times 10^{6}\right.$ cells/mice) were injected subcutaneously into the right flank of $\mathrm{C} 3 \mathrm{H}$ mice. Tumor volume and body weight were measured every 2 days. Tumor volume was calculated using the formula $\left(\mathrm{ab}^{2}\right) / 2$, in which a is the largest diameter and $\mathrm{b}$ is the shortest diameter of the tumor. Tumor weight was measured at the end of the treatment period. (A) Tumor volume, (B) tumor weight and $(\mathrm{C})$ body weight. The data are reported as mean \pm standard deviation of 6 mice. ${ }^{*} \mathrm{P}<0.05$ and ${ }^{* *} \mathrm{P}<0.01$, compared with the vehicle-treated control group.

tissues treated with vehicle. Furthermore, the percentage of TUNEL-positive apoptotic cells was significantly increased, with a significant increase in LC3B and reduction in p62 expression levels in apicidin-treated tumor tissues, compared with vehicle-treated control tumor tissues (Fig. 5B and C). These results demonstrated that apicidin effectively inhibited cell proliferation and HDAC8, and induced apoptosis and autophagy in vivo AT-84 mediated OSCC model. 


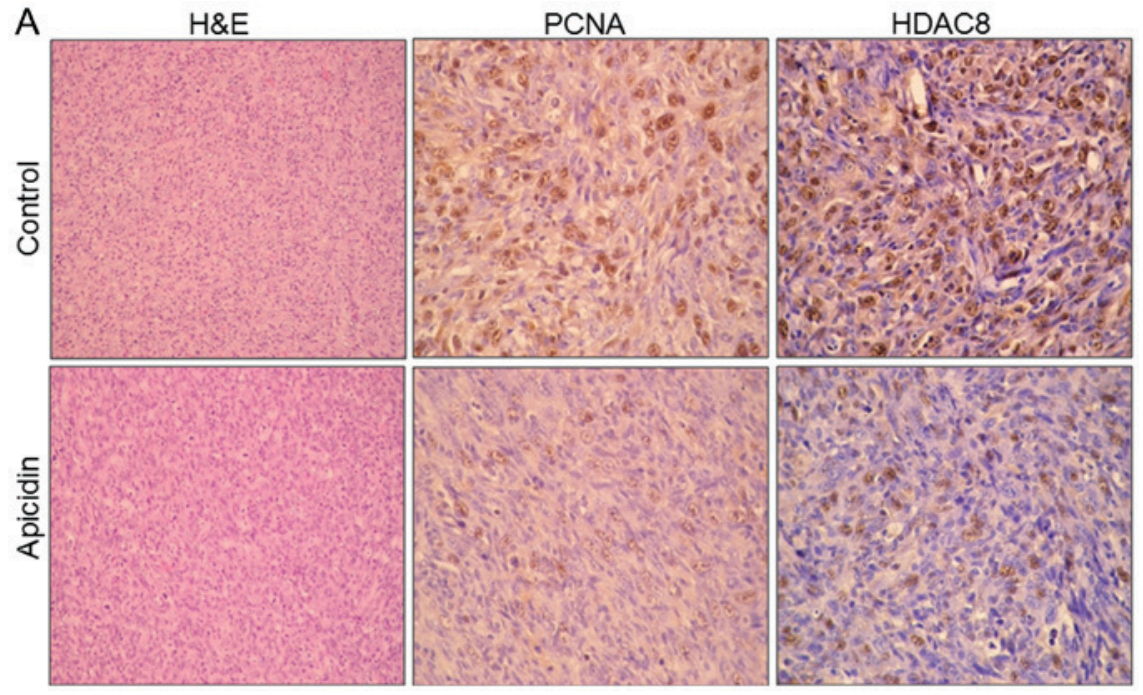

B

TUNEL

LC3B

p62
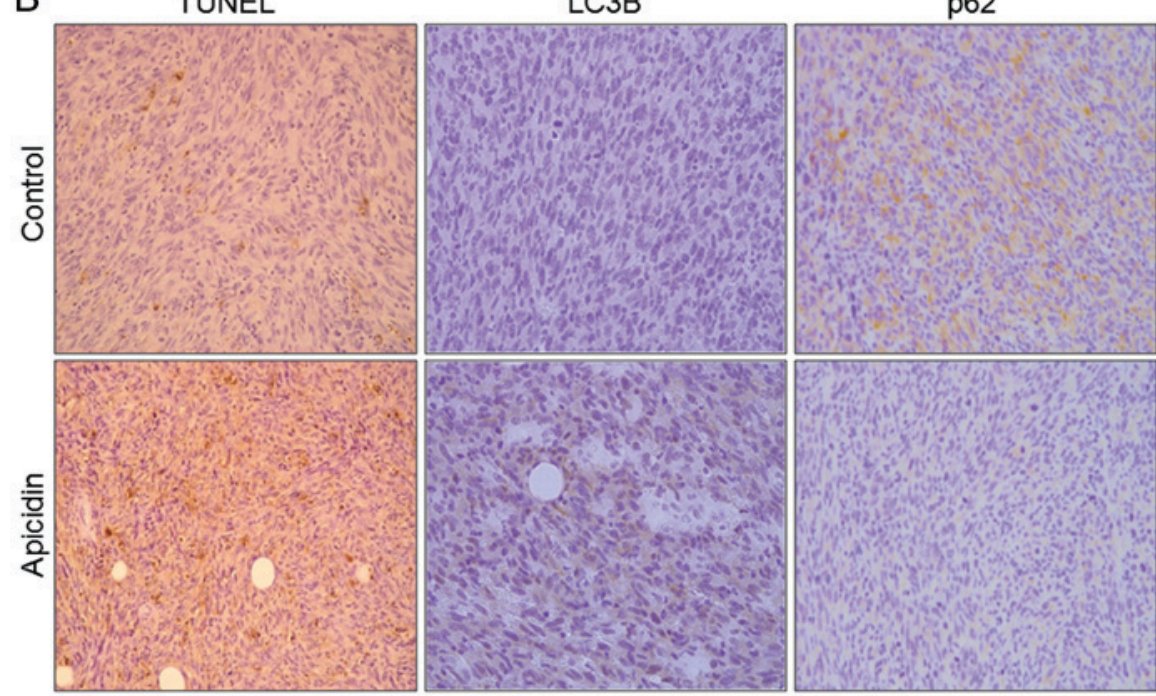

C
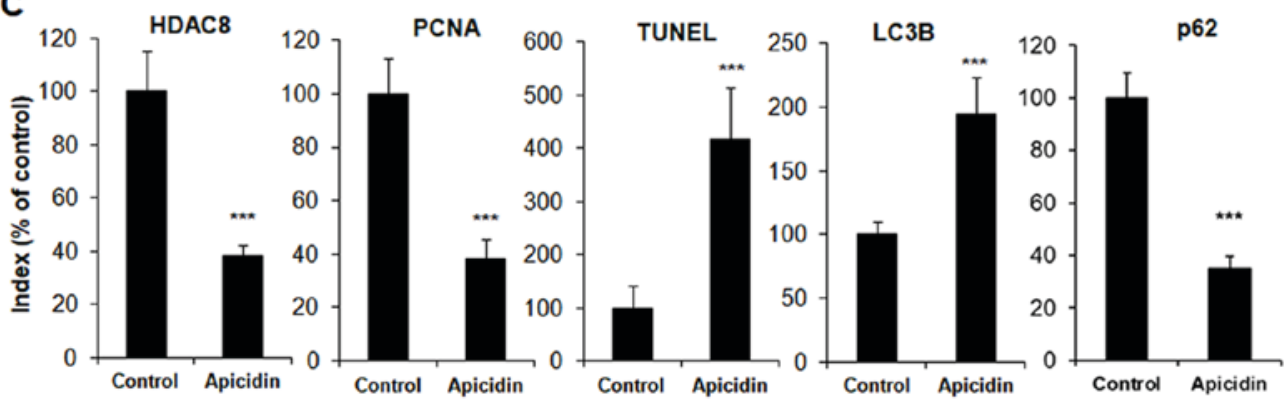

Figure 5. Effect of apicidin on PCNA, HDAC8, apoptosis and autophagy in AT-84 cell-derivated allografts. (A) H\&E staining of tumor sections (magnification, x200), and immunohistochemistry for PCNA and HDAC8 were performed on paraffin-embedded tumor sections (magnification, x400). (B) TUNEL assay and immunohistochemistry for LC3B and p62 were performed on paraffin-embedded tumor sections (magnification, x400). (C) Positive cells of immunostaining and TUNEL were counted, and the results are expressed as the mean \pm standard deviation. Five random fields from each experiment were counted under a microscope at maginification, $\mathrm{x} 400$. The index was calulated assuming that the amount of positive cells in the control was $100 \%{ }^{* * *} \mathrm{P}<0.001$, compared with the vehicle-treated control group. H\&E, hematoxylin and eosin; LC3B, microtubule associated protein 1 light chain 3B; PCNA, proliferating cell nuclear antigen; HDAC8, histone deacetylase 8; TUNEL, terminal deoxynucleotidyl transferase-mediated dUTP nick end labeling.

\section{Discussion}

Potential preclinical efficacy of HDAC inhibitors in oral cancer has been indicated, but there is limited knowledge regarding the therapeutic role of the HDAC inhibitor apicidin in $\operatorname{OSCC}(25,26)$. The present study investigated the antitumor effect of the HDAC inhibitor apicidin in murine OSCC. Inhibition of cell proliferation and the expression of HDAC were examined in the apicidin-treated AT- 84 cells. Apicidin significantly inhibited cell viability and induced histone acetylation in AT-84 cells, consistent with a previous study with human OSCC cell lines (20). As HDAC inhibitors do not 
inhibit all HDAC isoforms to the same extent, they may be grouped into pan HDAC inhibitors and class or iso-form selective HDAC inhibitors $(27,28)$. The development of selective HDAC inhibitors may be useful for effective chemotherapy. Apicidin is primarily known as a class I selective HDAC inhibitor; however, a number of studies have provided evidence that apicidin also inhibited class II HDACs $(10,29,30)$, and it is possible that specific HDAC inhibition by apicidin may have different effects depending on the cell or tissue type. To the best of our knowledge, isoform selective HDAC inhibition in apicidin treatment has not been previously reported in OSCC. HDAC2 was overexpressed in paraffin-embedded biopsies from patients with OSCC and increased HDAC6 expression was determined in tumor tissues, compared with normal oral squamous tissues, in previous reports $(31,32)$. In the results of the present study, no notable changes were determined in the levels of HDAC2 and HDAC6 expression by apicidin treatment in AT-84 cells. A recent study evaluated that HDAC8 overexpressed in human OSCC tissues and cell lines, and inhibition of HDAC8, could be an effective therapeutic tool for OSCC (5). The present results demonstrated that the level of HDAC8 expression was upregulated in the control, and apicidin notably inhibited HDAC8 expression in AT-84 cells. Although HDAC7 was also marginally reduced in the apicidin-treated AT-84 cells, compared with the control, the basal level of HDAC7 expression in the control was reduced, compared with other HDACs in AT-84 cells. Following these results, it was considered that HDAC8 inhibition by apicidin is highly associated with the recovery of histone acetylation and cell growth inhibition, rather than HDAC7 inhibition, in AT-84 cells. It was confirmed that HDAC8 was overexpressed in human and murine OSCC cell lines and apicidin also notably inhibited HDAC8 expression in human OSCC cell lines (data not shown). Therefore, the fact that apicidin inhibited HDAC8 may indicate that apicidin could be an efficient tumor-targeted drug for OSCC.

Subsequently, cell death induction by apicidin was examined in AT-84 cells. Apoptosis is the first identified programmed cell death process and its contribution to cancer research has been well documented (33). The present results demonstrated that apicidin significantly induced caspase-dependent apoptosis in AT-84 cells. Previous studies indicated that apicidin treatment primarily promoted apoptosis through an intrinsic caspase-dependent pathway in a number of cancer cells, in a manner similar to the present result $(20,34)$. Autophagy, a key homeostatic process in which cytosolic components are degraded and recycled through lysosomes, has been identified as a novel programmed cell death process. However, its role in cancer treatment remains controversial $(30,35)$. In the present study, formation of AVOs increased LC3B-II and ATG7 expression, and degradation of p62 indicated that apicidin notably induced autophagy in AT-84 cells. Although autophagy is considered a cell death mechanism, the current consensus is that the role of autophagy in cell death is primarily protective (36). Apicidin induced apoptosis and pro-survival autophagy in human OSCC cell lines (20). Additionally, the inhibition of HDAC8 induced autophagy as a protective function in human OSCC cells (5). In the present study, it was demonstrated that autophagy caused by apicidin may also have a protective effect on murine OSCC cells, although further confirmation is required. Overall, these results indicated that apicidin has an anti-proliferative effect and may induce apoptosis and autophagy in human and murine OSCC cell lines.

The in vivo anti-proliferative activity of apicidin has been reported in a number of cancer cell-derived tumor models, but, to the best of our knowledge, the in vivo antitumor effect of apicidin in OSCC has not been determined $(10,12,37)$. Subsequently, the antitumor effect of apicidin was evaluated using a tumor allograft mouse model, due to the in vitro effects of apicidin in OSCC cells. AT-84 cells induce OSCC in $\mathrm{C} 3 \mathrm{H}$ mice, and this animal model with AT- 84 cells is a commonly preferred syngeneic mouse model, which is realistic and efficient for oral cancer experiments (38). The present results demonstrated that apicidin notably inhibited AT-84 cell-derived tumor growth in the animal model. Evaluation of cell proliferation in tissues of experimental animals is essential for carcinogenesis and the most common method to identify proliferating cells in tissue sections is immunohistochemical detection of PCNA (39). Elevated expression of PCNA has been observed in the buccal tissues of a 7,12-dimethylbenz(a) anthracene-induced oral cancer animal model (40). PCNA has been demonstrated to be associated with the prognosis in a number of cancer types, including oral cancer (41). This anti-proliferative activity was confirmed by a significant decrease in PNCA expression in the apicidin-treated group, compared with the vehicle-treated control group. In the immunohistochemical examinations, apicidin treatment increased the percentage of TUNEL-positive apoptotic cells, and reduced the expression levels of LC3B and p62, compared with the vehicle-treated control group. These results indicated that apicidin induced apoptosis and autophagy in tumor tissues, which is similar to the in vitro results. HDACs are considered to localize in the nucleus, in order to regulate gene transcription (42). Class I HDACs are localized exclusively in the nucleus, whereas class II HDACs are larger proteins that are shuttled between the cytoplasm and the nucleus $(43,44)$. However, previous studies have indicated that the subcellular localization of class I HDACs can be located within the nucleus as well as within the cytoplasm, and translocalization of HDACs may be associated with the transcriptional activity of these HDACs on histone or non-histone proteins $(5,44,45)$. In the present study, strong HDAC8 expression in AT-84 cell-derived tumor tissues was observed in the nucleus and cytoplasm. In particular, apicidin treatment significantly inhibited the level of HDAC8 expression in the nuclear compartments of tumor tissues, compared with the vehicle group. Consistent with this result, in a number of cancer types, including esophageal squamous cell carcinoma, the localization of HDAC8 in the nucleus and cytoplasm has been detected (46). However, a previous study demonstrated that overexpression of HDAC8 was primarily observed in cytoplasmic compartments in human OSCC tissues from patients (5). It was demonstrated that subcellular localization of HDAC8 may be dynamically regulated depending on the intended function or cellular condition, and further evaluation of HDAC8 localization in OSCC is required. Overall, these results indicated that apicidin effectively inhibited cancer cells growth and HDAC8 expression, in vitro and in vivo, in murine OSCC cells.

In conclusion, the present study demonstrated that the HDAC inhibitor apicidin exhibits antitumor activity in vitro and in vivo in murine OSCC, resulting in the inhibition of cell 
proliferation and the induction of apoptosis and autophagy. Upregulated HDAC8 expression, a novel therapeutic target of OSCC, was selectively inhibited by apicidin treatment in murine OSCC. Collectively, these data may contribute to understanding the antitumor mechanism of apicidin in OSCC and indicate the potential of apicidin as a therapeutic agent against OSCC.

\section{Acknowledgements}

Not applicable.

\section{Funding}

The present study was supported by the Basic Science Research Program through the National Research Foundation of Korea funded by the Ministry of Education, Science and Technology (grant nos. NRF-2011-0023907 and NRF-2016R1D1A3B03931034).

\section{Availability of data and materials}

All data generated or analyzed during this study are included in this published article.

\section{Authors' contributions}

MYA conducted all the experiments in the present study.

\section{Ethics approval and consent to participate}

All experiments were performed under protocols approved by the Ethical Committee of Animal Care and Use at Pusan National University Hospital (approval no. PNUH-2016-090).

\section{Patient consent for publication}

Not applicable.

\section{Competing interests}

The author declares that they have no competing interests.

\section{References}

1. Manal M, Chandrasekar MJ, Gomathi Priya J and Nanjan MJ: Inhibitors of histone deacetylase as antitumor agents: A critical review. Bioorg Chem 67: 18-42, 2016.

2. Dokmanovic M, Clarke C and Marks PA: Histone deacetylase inhibitors: Overview and perspectives. Mol Cancer Res 5: 981-989, 2007.

3. Iizuka M and Smith MM: Functional consequences of histone modifications. Curr Opin Genet Dev 13: 154-160, 2003.

4. Marks P, Rifkind RA, Richon VM, Breslow R, Miller T and Kelly WK: Histone deacetylases and cancer: Causes and therapies. Nat Rev Cancer 1: 194-202, 2001.

5. Ahn MY and Yoon JH: Histone deacetylase 8 as a novel therapeutic target in oral squamous cell carcinoma. Oncol Rep 37: 540-546, 2017.

6. Kim HJ and Bae SC: Histone deacetylase inhibitors: Molecular mechanisms of action and clinical trials as anti-cancer drugs. Am J Transl Res 3: 166-179, 2011.

7. Batty N, Malouf GG and Issa JP: Histone deacetylase inhibitors as anti-neoplastic agents. Cancer Lett 280: 192-200, 2009.

8. Grant S and Dai Y: Histone deacetylase inhibitors and rational combination therapies. Adv Cancer Res 116: 199-237, 2012.
9. Bauden M, Tassidis $\mathrm{H}$ and Ansari D: In vitro cytotoxicity evaluation of HDAC inhibitor Apicidin in pancreatic carcinoma cells subsequent time and dose dependent treatment. Toxicol Lett 236: 8-15, 2015.

10. Ahn MY, Kang DO, Na YJ, Yoon S, Choi WS, Kang KW, Chung HY, Jung JH, Min do S and Kim HS: Histone deacetylase inhibitor, apicidin, inhibits human ovarian cancer cell migration via class II histone deacetylase 4 silencing. Cancer Lett 325: 189-199, 2012.

11. Cheong JW, Chong SY, Kim JY, Eom JI, Jeung HK, Maeng HY, Lee ST and Min YH: Induction of apoptosis by apicidin, a histone deacetylase inhibitor, via the activation of mitochondria-dependent caspase cascades in human Bcr-Abl-positive leukemia cells. Clin Cancer Res 9: 5018-5027, 2003.

12. Lai JP, Sandhu DS, Moser CD, Cazanave SC, Oseini AM, Shire AM, Shridhar V, Sanderson SO and Roberts LR: Additive effect of apicidin and doxorubicin in sulfatase 1 expressing hepatocellular carcinoma in vitro and in vivo. J Hepatol 50: 1112-1121, 2009.

13. Zhang J, Lai Z, Huang W, Ling H, Lin M, Tang S, Liu Y and Tao Y: Apicidin inhibited proliferation and invasion and induced apoptosis via mitochondrial pathway in non-small cell lung cancer GLC-82 cells. Anticancer Agents Med Chem 17: 1374-1382, 2017.

14. Bauden M, Tassidis $H$ and Ansari D: In vitro cytotoxicity evaluation of HDAC inhibitor Apicidin in pancreatic carcinoma cells subsequent time and dose dependent treatment. Toxicol Lett 236: 8-15, 2015.

15. Ahn MY, Ahn JW, Kim HS, Lee J and Yoon JH: Apicidin inhibits cell growth by downregulating IGF-1R in salivary mucoepidermoid carcinoma cells. Oncol Rep 33: 1899-1907, 2015.

16. Keleş E, Lianeri M and Jagodziński PP: Apicidin suppresses transcription of 17 $\beta$-hydroxysteroid dehydrogenase type 1 in endometrial adenocarcinoma cells. Mol Biol Rep 38: 3355-3360, 2011.

17. Markopoulos AK: Current aspects on oral squamous cell carcinoma. Open Dent J 6: 126-130, 2012.

18. Cheng YS, Rees T and Wright J: A review of research on salivary biomarkers for oral cancer detection. Clin Transl Med 3: 3, 2014.

19. Park JW, Kim CH, Ha YC, Kim MY and Park SM: Count of platelet and mean platelet volume score: Serologic prognostic factor in patients with oral squamous cell carcinoma. J Korean Assoc Oral Maxillofac Surg 43: 305-311, 2017.

20. Ahn MY, Ahn SG and Yoon JH: Apicidin, a histone deaceylase inhibitor, induces both apoptosis and autophagy in human oral squamous carcinoma cells. Oral Oncol 47: 1032-1038, 2011.

21. Lou E, Kellman RM, Hutchison R and Shillitoe EJ: Clinical and pathological features of the murine AT- 84 orthotopic model of oral cancer. Oral Dis 9: 305-312, 2003.

22. Schultz-Hector S and Haghayegh S: Beta-fibroblast growth factor expression in human and murine squamous cell carcinomas and its relationship to regional endothelial cell proliferation. Cancer Res 53: 1444-1449, 1993.

23. Hier MP, Black MJ, Shenouda G, Sadeghi N and Karp S: A murine model for the immunotherapy of head and neck squamous cell carcinoma. Laryngoscope 105: 1077-1080, 1995.

24. Pang S, Kang MK, Kung S, Yu D, Lee A, Poon B, Chen IS, Lindemann B and Park NH: Anticancer effect of a lentiviral vector capable of expressing HIV-1 Vpr. Clin Cancer Res 7: 3567-3573, 2001.

25. Murakami J, Asaumi J, Maki Y, Tsujigiwa H, Kuroda M, Nagai N, Yanagi Y, Inoue T, Kawasaki S, Tanaka N, et al: Effects of demethylating agent 5-aza-2(')-deoxycytidine and histone deacetylase inhibitor FR901228 on maspin gene expression in oral cancer cell lines. Oral Oncol 40: 597-603, 2004.

26. Chung YL, Lee MY and Pui NN: Epigenetic therapy using the histone deacetylase inhibitor for increasing therapeutic gain in oral cancer: Prevention of radiation-induced oral mucositis and inhibition of chemical-induced oral carcinogenesis. Carcinogenesis 30: 1387-1397, 2009.

27. Rikiishi H: Autophagic and apoptotic effects of HDAC inhibitors on cancer cells. J Biomed Biotechnol 2011: 830260, 2011.

28. Balasubramanian S, Verner E and Buggy JJ: Isoform-specific histone deacetylase inhibitors: The next step? Cancer Lett 280: 211-221, 2009.

29. Kim SN, Choi HY and Kim YK: Regulation of adipocyte differentiation by histone deacetylase inhibitors. Arch Pharm Res 32: 535-541, 2009.

30. Ahn MY and Yoon JH: Histone deacetylase 7 silencing induces apoptosis and autophagy in salivary mucoepidermoid carcinoma cells. J Oral Pathol Med 46: 276-283, 2017. 
31. Chang HH, Chiang CP, Hung HC, Lin CY, Deng YT and Kuo MY: Histone deacetylase 2 expression predicts poorer prognosis in oral cancer patients. Oral Oncol 45: 610-614, 2009.

32. Sakuma T, Uzawa K, Onda T, Shiiba M, Yokoe H, Shibahara T and Tanzawa H. Aberrant expression of histone deacetylase 6 in oral squamous cell carcinoma. Int J Oncol 29: 117-124, 2006.

33. Eisenberg-Lerner A, Bialik S, Simon HU and Kimchi A: Life and death partners: Apoptosis, autophagy and the cross-talk between them. Cell Death Differ 16: 966-975, 2009.

34. Ahn MY, Lee J, Na YJ, Choi WS, Lee BM, Kang KW and Kim HS: Mechanism of apicidin-induced cell cycle arrest and apoptosis in Ishikawa human endometrial cancer cells. Chem Biol Interact 179: 169-177, 2009.

35. Alirezaei M, Kemball CC, Flynn CT, Wood MR, Whitton JL and Kiosses WB: Short-term fasting induces profound neuronal autophagy. Autophagy 6: 702-710, 2010.

36. Yonekawa T and Thorburn A: Autophagy and cell death. Essays Biochem 55: 105-117, 2013.

37. Ahn MY, Chung HY, Choi WS, Lee BM, Yoon S and Kim HS Anti-tumor effect of apicidin on Ishikawa human endometrial cancer cells both in vitro and in vivo by blocking histone deacetylase 3 and 4. Int J Oncol 36: 125-131, 2010.

38. Nair DV and Reddy AG: Mouse models of oral cancer: Challenges and opportunities. Int J Adv Biol Res 7: 203-207, 2017.

39. Muskhelishvili L, Latendresse JR, Kodell RL and Henderson EB: Evaluation of cell proliferation in rat tissues with BrdU, PCNA, Ki-67(MIB-5) immunohistochemistry and in situ hybridization for histone mRNA. J Histochem Cytochem 51: 1681-1688, 2003.
40. Velu P, Vinothkumar V, Babukumar S and Ramachandhiran D: Chemopreventive effect of syringic acid on 7,12-dimethylbenz(a) anthracene induced hamster buccal pouch carcinogenesis. Toxicol Mech Methods 27: 631-640, 2017.

41. Saraç S, Ayhan A, Hosal AS and Kaya S: Prognostic significance of PCNA expression in laryngeal cancer. Arch Otolaryngol Head Neck Surg 124: 1321-1324, 1998.

42. Guo X, Ruan H, Li X, Qin L, Tao Y, Qi X, Gao J, Gan L, Duan S and Shen W: Subcellular localization of class I histone deacetylases in the developing xenopus tectum. Front Cell Neurosci 9: $510,2016$.

43. Li Y, Shin D and Kwon SH: Histone deacetylase 6 plays a role as a distinct regulator of diverse cellular processes. FEBS J 280: 775-793, 2013.

44. Yang WM, Tsai SC, Wen YD, Fejer G and Seto E: Functional domains of histone deacetylase-3. J Biol Chem 277: 9447-9454, 2002.

45. Grozinger CM and Schreiber SL: Regulation of histone deacetylase 4 and 5 and transcriptional activity by 14-3-3-dependent cellular localization. Proc Natl Acad Sci USA 97: 7835-7840, 2000.

46. Nakagawa M, Oda Y, Eguchi T, Aishima S, Yao T, Hosoi F, Basaki Y, Ono M, Kuwano M, Tanaka M and Tsuneyoshi M: Expression profile of class I histone deacetylases in human cancer tissues. Oncol Rep 18: 769-774, 2007.

(i) (5) This work is licensed under a Creative Commons cc) Attribution-NonCommercial-NoDerivatives 4.0 International (CC BY-NC-ND 4.0) License. 